\title{
Patientenorientierte stationäre Kooperation zwischen Ärzten und Pflegekräften - Behinderungen und Voraussetzungen
}

\author{
Karin Rausch
}

Online publiziert: 24. Oktober 2012

(C) Springer-Verlag Wien 2012

Ergebnisse einer Pilotstudie in 3 Krankenhäusern (Organisationsanalyse und qualitative Inhaltsanalyse verschrifteter Tonbandinterviews mit 48 medizinischen und pflegerischen Leitungskräften; Auswertung nach Konfliktmustern und -konstellationen in der Kooperation) zeigten eine für gelingende stationäre Kooperation hinderliche Organisationsstruktur und mangelnden Bezug beider Kooperationspartner aufeinander: Versäulung nach Berufsgruppen, divergierende Arbeitskontexte, Berufsgruppenkulturen und Zeitmanagements, inkompatible Hierarchien, zu komplexe Anweisungsstruktur, vor allem keine gemeinsame Orientierung auf gelingende Kooperation. Die Betroffenen verbrauchten viel Zeit und Energien auf störanfälliges Arbeiten unter Dauerkonflikten. Es gab kaum Erfahrungen mit Konfliktlösungen, wie auch kaum miteinander gesprochen wurde.
Im Interesse von ökonomischer Effizienz und Fehlervermeidung müssen am gewünschten Ergebnis orientierte reibungsarme Arbeitsabläufe mit dem Ziel gelingender Kooperation organisiert werden; ggfs. mit einem Prozessmanager.

Dazu bedarf es der Erweiterung des Wissens übereinander, Respekt und Wertschätzung für die jeweiligen Arbeitsbeiträge, Vereinfachung der Anweisungsstruktur, Passung der Zeitmanagements und gemeinsames prozessorientiertes Planen und Handeln.

Den Betroffenen ist zu wünschen, dass sie die Erfahrung machen: Gelingende Zusammenarbeit kann Spaß machen.

K. Rausch $(\bowtie)$

Hannover, Deutschland 